\title{
Modelo de Interoperabilidade para Utilização dos Recursos dos Ambientes Virtuais de Aprendizagem Através dos Dispositivos Móveis
}

\author{
Kleber Tavares Fernandes ${ }^{1}$, Márcia Jacyntha N. R. Lucena ${ }^{1}$, Apuena V. Gomes ${ }^{3}$ \\ ${ }^{1}$ Departamento de Informática e Matemática Aplicada \\ Universidade Federal do Rio Grande do Norte (UFRN) \\ 59078-970 - Natal, RN - Brasil \\ ${ }^{2}$ Instituto Metrópole Digital \\ Universidade Federal do Rio Grande do Norte (UFRN) \\ 59078-970 - Natal, RN - Brasil \\ Kleber76@gmail.com.br, marciaj@dimap.ufrn.br, apuena@imd.ufrn.br
}

\begin{abstract}
The technological evolution has been making the Distance Education accessible for a greater number of citizens anytime and anywhere. The potential increase of the supply for mobile devices integrated to mobile learning environments allows that the information comes out of the physical environment, creating opportunities for students and teachers to create geographically distributed learning scenarios. However, many applications developed for these environments remain isolated from each other and do not become integrated sufficiently into the virtual learning environments (AVA). This paper presents an interoperability model between mobile devices and distinct AVA based on webservices.
\end{abstract}

Resumo. A evolução tecnológica tem tornado a Educação a Distância acessivel para um maior número de cidadãos em qualquer hora e em qualquer lugar. O aumento potencial da oferta de dispositivos móveis integrados a ambientes de aprendizado móvel permite que a informação saia dos ambientes fisicos das instituições de ensino, oportunizando a alunos e professores criarem cenários de aprendizagem geograficamente distribuídos. Entretanto, muitos dos aplicativos desenvolvidos para estes ambientes ainda permanecem isolados uns dos outros e não se integram de maneira suficiente aos ambientes virtuais de aprendizagem (AVA). Este artigo apresenta um modelo de interoperabilidade entre dispositivos móveis e AVA distintos baseado em webservices.

\section{Introdução}

Com a evolução tecnológica e a globalização mundial, as instituições educacionais brasileiras, principalmente as de nível superior, vêm passando por um processo de mudança, com relação a Educação a Distancia $(\mathrm{EaD})$ no processo educacional. Este cenário tem oportunizado o acesso a educação para um maior número de cidadãos que, 
devido a diversos fatores, não tinham como concluir seus estudos, principalmente no que se refere ao acesso ao ensino superior. Segundo Rodrigues (2011), o processo de ensino-aprendizagem, no contexto de $\mathrm{EaD}$, tem sido influenciado pelas novas tecnologias. Uma vez que professores e alunos estão separados no tempo e no espaço, a evolução tecnológica mostra-se determinante. Podemos afirmar que a EaD é mais flexível por não limitar o aluno aos locais e horários determinados pelas Instituições.

No aspecto do uso das tecnologias de informação e comunicação para promover a $\mathrm{EaD}$, algumas destas instituições adotaram como plataformas de ensino, seus Ambientes Virtuais de Aprendizagem (AVA) com o objetivo de oferecer suporte a esta modalidade de ensino, utilizando-se de estratégias e recursos variados, sejam eles para apresentação de conteúdos, comunicação, interatividade e avaliação. Os AVA promovem a aprendizagem do aluno através do estímulo à construção do seu conhecimento a partir da sua prática, reflexões, interações e uso dos diferentes recursos e mídias. Estes ambientes virtuais são sistemas integradores e abrangentes capazes de organizar e definir um ponto focal para os processos educacionais mediados por computador, apoiar os professores nas atividades de acompanhamento e promover o engajamento dos alunos. A interação dos alunos com o ambiente e com os conteúdos disponíveis no ambiente torna-se fundamental para que os alunos possam organizar suas ideias, compartilhar seus conhecimentos tornando-se sujeitos autônomos de sua aprendizagem [Penteado, 2013]. Os AVA atuam como ferramentas para EaD e em complementação ao conteúdo dado em sala de aula, otimizam a assistência entre professores e alunos.

Com o advento das tecnologias móveis surge o acesso à informação em tempo real, em qualquer hora e em qualquer ponto geográfico. Os dispositivos móveis (tablets e smartphones) oferecem facilidade de acesso à Internet, de maneira simples, rápida e agradável. Com os avanços e aumento do potencial dessas tecnologias surge o mobile learning ou m-Learning. Para Bottentuit (2012) Mobile learning, aprendizagem móvel ou aprendizagem com mobilidade, é um conceito criado para a aprendizagem que utiliza recursos móveis, ou seja, equipamentos e dispositivos que permitam ao aprendiz a locomoção enquanto acessam o conteúdo. $\mathrm{O}$ m-Learning passa a permitir que a informação e/ou o conhecimento saiam dos ambientes físicos das instituições com maior celeridade e conquistem outros espaços, em diferentes tempos e momentos da vida (casa, trabalho, entre outros), oferecendo mais uma forma de acesso. Segundo Yau e Joy (2010) este meio de ensino permite que estudantes e professores possam usufruir dos recursos oferecidos pelas tecnologias móveis.

Nesse sentido parece natural que os AVA possam ser acessados através de dispositivos móveis formando um ambiente de m-learning. Os aplicativos voltados para esse propósito devem acessar de maneira transparente um ou mais recursos dos AVA, antes disponíveis apenas em ambientes desktop. Porém, é importante observar as necessidades apontadas por Silva et al (2011) em disponibilizar conteúdos de forma apropriada aos dispositivos móveis. Algumas restrições de hardware como por exemplo: tamanho reduzido de tela, baixo poder de processamento, capacidade limitada de armazenamento, comunicação com taxas de transmissão geralmente menores do que as das redes fixas, adequação a aspectos de usabilidade, carência de padrões arquiteturais e baixa autonomia da bateria podem influenciar negativamente na mobilidade do dispositivo e, até mesmo, na experiência do usuário no uso das interfaces de m-learning. 
Este trabalho apresenta um modelo de interoperabilidade entre dispositivos móveis e ambientes virtuais de aprendizagem, utilizando técnicas computacionais, de engenharia e arquitetura de software que proporcione a comunicação entre um aplicativo móvel e os AVA, formando um ambiente de m-learning.

Nossa intenção com este trabalho não é apresentar todos os recursos e modelos utilizados para promover a interoperabilidade entre dispositivos móveis e AVA, uma vez que, outros poderão surgir com os avanços tecnológicos. O objetivo do trabalho é oferecer uma solução viável de comunicação entre estes sistemas, que venha a favorecer o processo ensino e aprendizagem à distância.

Como contribuição para o cenário educacional, esta proposta vem se configurar em mais uma possibilidade de interação entre professor e aluno. $O$ caráter não presencial rompe possíveis "barreiras geográficas", quando permite que o aluno acesse ambientes virtuais em qualquer lugar e tempo e que o professor acompanhe seus os avanços e dificuldades.

Este artigo está organizado da seguinte maneira: a Seção 2 apresenta os aspectos da mobilidade na educação a distância; a Seção 3 descreve as tecnologias utilizadas para promover a interoperabilidade entre sistemas; a Seção 4 apresenta o modelo de interoperabilidade entre um APP e um AVA; a Seção 5 apresenta considerações finais e trabalhos futuros.

\section{Aspectos da mobilidade na educação a distância}

Perrenoud (2000) define as dez novas competências para ensinar, entre elas a competência oito ("Utilizar novas tecnologias") que propõe o uso da Tecnologia da Informação e Comunicação (TIC) em prol do ensino. De fato, as TIC têm contribuído para transformação na maneira de estudar, ensinar, comunicar, trabalhar e de pensar. Soma-se a isso o advento da mobilidade tecnológica e da Internet que trouxeram a possibilidade de ensinar e aprender independentemente do espaço, seja físico ou temporal.

O uso de dispositivos móveis amplia as possibilidades de ensino sem limites geográficos e temporais. Essa prática chamada de mobile learning (m-learning) possibilita o acesso a informações e a interação professor aluno através das tecnologias mobile. Para Bowker (2000) e Koschembahr (2005) o m-Learning é definido como processos de aprendizagem que ocorrem, necessariamente apoiados pelo uso de tecnologias de informação móveis e que têm como característica fundamental a mobilidade de atores humanos, que podem estar fisicamente/geograficamente distante de outros atores e também de espaços físicos formais de educação, tais como salas de aula, salas de treinamento ou local de trabalho.

O m-learning é caracterizado pela sua capacidade de proporcionar forte interação entre os alunos e professores, possibilitando a contribuição, participação e acesso ao ambiente de aprendizagem por meio de quaisquer dispositivos móveis a qualquer momento em qualquer lugar. Tais dispositivos utilizados nesta interação, por si só ou em combinação com outras tecnologias da informação e da comunicação, 
permitem uma aprendizagem desdobrada em vários formatos, assim como, apoia objetivos educacionais, administrativos ou de gestão [Freitas e Barbosa, 2012].

Georgieva, Smrikarov e Georgiev (2011) afirmam que o m-learning é uma nova tendência no desenvolvimento do e-learning, na qual os dispositivos móveis auxiliam os estudantes no acesso aos materiais educativos em qualquer hora e lugar. Este aspeto é importante para as Instituições de Ensino, porque professores e alunos chegam a suas salas de aula com esses dispositivos móveis, portáteis e conectados à Internet, à mão.

Devido à evolução dos dispositivos móveis, sua versatilidade e mobilidade, aplicações que eram conhecidas apenas em ambientes desktop, passaram a ser disponibilizadas neste novo tipo de plataforma. Como vantagens, o m-Learning possibilita o acesso às informações através de um dispositivo fácil de transportar e de fácil uso, ou seja, estão permanentemente acessíveis aos utilizadores.

O uso das tecnologias educacionais móveis ainda depende de muitas variáveis alheias ao professor, gestor ou escola, mas cabe a eles transformar em aprendizagem eficaz o uso destes recursos e às políticas públicas estimular a adoção de tecnologias diversificadas, oferecer resoluções técnicas e formação permanente dos educadores [Dannemann, 2013].

\section{Tecnologias utilizadas para promover a interoperabilidade entre sistemas}

Nesta seção apresentamos as tecnologias ligadas ao desenvolvimento do Middleware (MW) e WebService (WS) que são utilizadas neste trabalho.

\subsection{Middleware}

Para Coulouris (2005) o middleware "é um termo aplicado a uma camada de software que fornece uma abstração da programação, assim como o mascaramento de heterogeneidade das redes, do hardware, de sistemas operacionais e linguagens de programação subjacentes". Tal camada configura funções de identificação, autorização, autenticação, segurança, entre outras. O middleware implementa a comunicação e o compartilhamento de recursos e aplicativos distribuídos.

Segundo Mahmoud (2004) "o papel do middleware em sistemas de informação será cada vez mais importante, especialmente em áreas tecnológicas emergentes, como a computação móvel onde a integração de aplicações e serviços a partir de redes sem fio se torna cada vez mais necessária devido a expansão da tecnologia”.

Um middleware forma uma camada entre aplicações e plataformas distribuídas cuja finalidade é proporcionar um grau de transparência de distribuição. [Tanenbaum, 2007]

Neste trabalho pretende-se fazer middleware de modo que sejam simples as configurações, adaptações e personalizações da aplicação conforme sejam necessárias. 


\subsection{Webservice}

Com o surgimento da comunicação através das redes de computadores e da necessidade de diferentes softwares se comunicarem entre si surgiu o conceito de Webservice (WS).

Os Webservices permitem que sistemas desenvolvidos em várias linguagens, sendo executados em diversas plataformas, transmitam e recebam informações padronizadas entre si, permitindo uma interação entre os dispositivos, mais abrangente que qualquer outra tecnologia de computação distribuída existente.

Segundo Kalin (2009) Webservice é um tipo de aplicação para web distribuída, cujos componentes podem ser aplicados e executados em dispositivos distintos. Configura-se como um mecanismo de comunicação que permite a interoperabilidade entre sistemas. Para Kreger (2001) um Webservice descreve uma coleção de operações que são acessíveis pela rede através de mensagens XML (eXtensible Markup Language) padronizadas. Porém existe a tecnologia JSON (Javascript Object Notation) que também possibilita a troca de mensagens de forma mais leve. O uso de Webservice possibilita que aplicações desenvolvidas em plataformas e linguagens diversificadas troquem informações padronizadas permitindo a interação entre elas com rapidez, facilidade e baixo custo, conforme ilustrado na Figura 1.

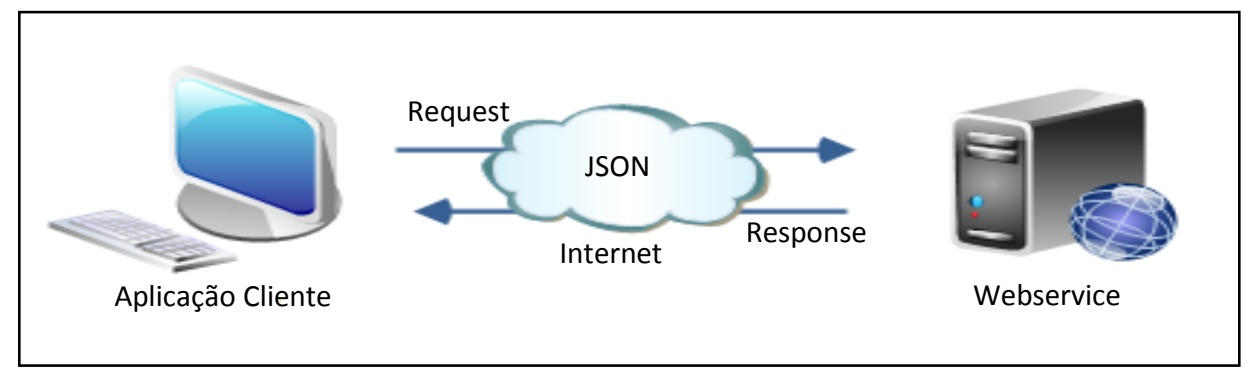

Figura 1: Troca de informações entre uma aplicação cliente e um WebService.

Souza (2004) explica que os Webservices utilizam tecnologias que permitem que os serviços sejam disponibilizados pela WEB transportando e transformando dados entre aplicações com base em XML ou JSON. Nesse contexto, a XML e JSON além de funcionar como um padrão para troca de mensagens também têm o papel de definir os serviços. Sua sintaxe especifica como os dados são representados, transmitidos e detalhes de como são publicados e descobertos.

\section{Modelo de interoperabilidade entre APP e AVA}

Nesta sessão, será descrita a nossa proposta de um modelo para promover a integração de uma aplicação no domínio da educação à distância, executada através dos dispositivos móveis, e que utiliza os recursos de questionário dos AVA.

A função de um middleware é estabelecer a comunicação entre aplicações que não se comunicam diretamente. Para o APP o uso de um middleware é de fundamental importância, pois o torna flexível para novas possibilidades de comunicação com outras funcionalidades dos AVA, além da possibilidade de registrar o log de transações realizadas entre as partes e outras questões relacionadas à segurança. $\mathrm{O}$ middleware foi 
desenvolvido baseado num padrão de interoperabilidade que permite a comunicação com diversos AVA. A figura 2 representa o diagrama da estratégia de comunicação que foi desenvolvida em duas etapas: entre o APP e o Middleware via Web Services e entre o Middleware e o AVA via Web Services distintos.

Para a troca de informações adotou-se o JSON (Java Script Object Notation). O JSON é um formato de interconexão de dados utilizado em ambientes cliente-servidor que possibilita o desenvolvimento nas mais variadas linguagens. O JSON atualmente vem sendo utilizado como linguagem padrão para comunicação entre sistemas nas mais diversas plataformas. A disponibilidade de bibliotecas e o desempenho proporcionado pela simplicidade da transmissão de dados utilizando JSON influenciaram na decisão por utilizá-lo.

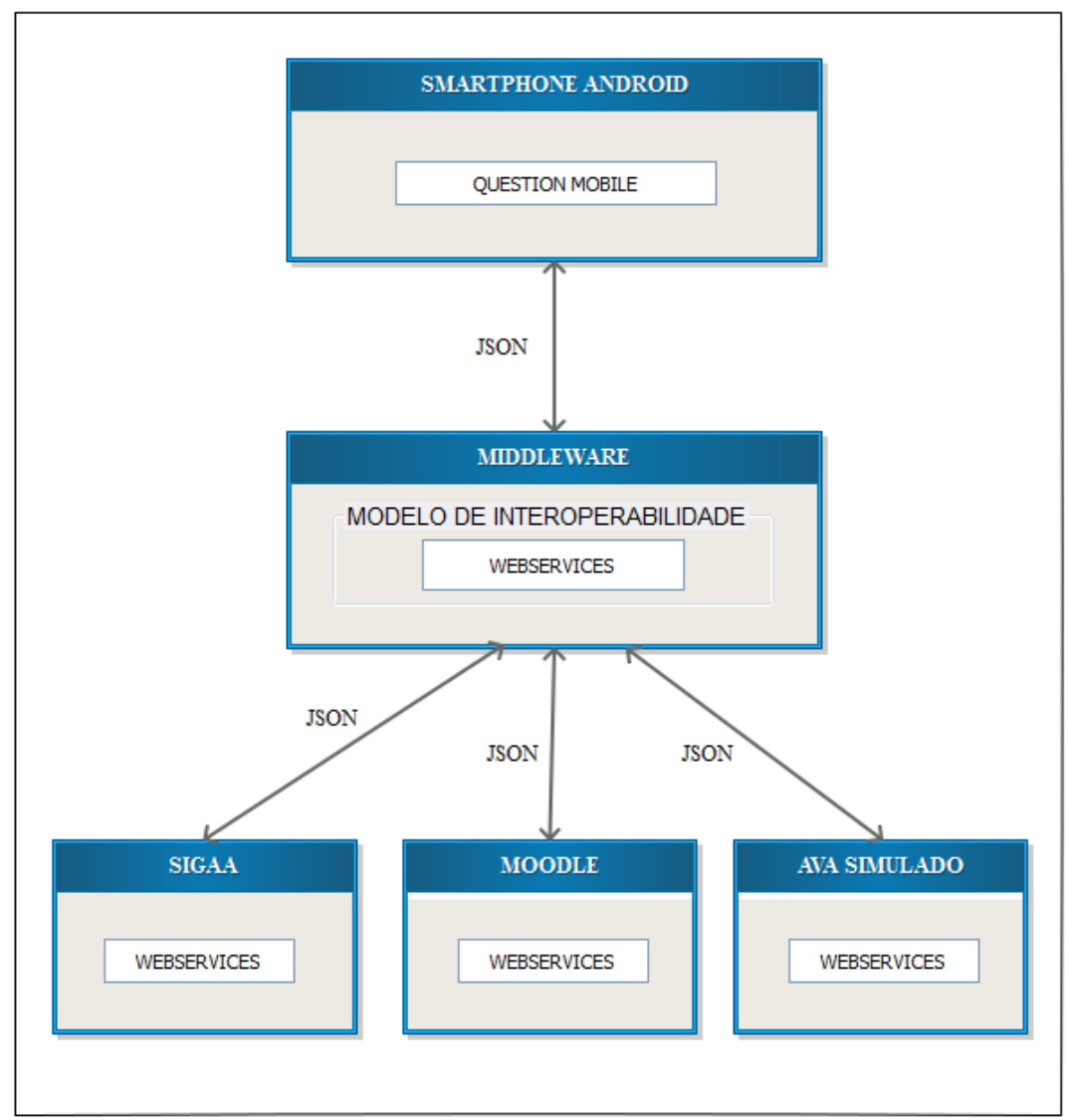

Figura 2: Modelo de interoperabilidade.

A interoperabilidade entre o APP e os AVA, conforme ilustrado na Figura 2, é realizado da seguinte forma:

- O APP é executado no Smartphone do usuário;

- O APP se conecta ao servidor do middleware através da URL cadastrada pelo usuário ou através do serviço de consulta ao MW que fornecerá os AVA ao APP; 
- O middleware disponibiliza o módulo com o padrão de comunicação de dados necessários à conexão com os diversos AVA;

- O middleware se conecta ao AVA selecionado pelo usuário.

Para que funcione adequadamente o AVA precisará implementar os serviços baseados no padrão estabelecido pelo modelo de interoperabilidade para que o middleware consiga se conectar a ele e interagir com os questionários armazenados em seu banco de dados.

Desta maneira, o QM apresenta uma estratégia de comunicação que é a utilização de WebServices padronizados pelo middleware.

O middleware poderá ser executado em um servidor de aplicação. Este servidor é responsável por estabelecer a comunicação com os AVA que desejem utilizar o APP. A desvantagem é que recursos computacionais de um servidor serão alocados para o middleware, porém como vantagens teremos a flexibilidade, melhoria no desempenho e na confiabilidade, assim como a manutenibilidade e disponibilidade do serviço.

Por questões de padronização e garantia de comunicação, implementou-se uma biblioteca para troca de dados entre o APP e os AVA. A biblioteca contém a descrição dos formatos de requisição e resposta de todos os serviços (tipo dos dados, campos obrigatórios, constantes, entre outros) e os métodos de validação dos dados. O middleware se responsabiliza por saber qual serviço será consumido baseado na escolha do usuário através do APP.

O desenvolvimento de uma única aplicação móvel que faz a conexão diretamente com os AVA traria o inconveniente de o usuário instalar novas versões do APP à medida que este fosse adaptado aos novos AVA. Além disso, a implementação e uso do MW desonera o consumo da bateria e o processamento do aparelho que esteja executando o APP, ampliando sua performance.

O modelo de interoperabilidade propõe a implementação e uso de seis serviços através dos quais será possível realizar diversas operações de troca de informações. Todos os serviços têm um só parâmetro de entrada (request) e um só parâmetro de saída (response) de tipos que encapsulam as informações necessárias para realizar as requisições e o resultado da operação. Os serviços são descritos a seguir:

DoLogin: Operação destinada a realizar login no AVA, ou seja, criar uma sessão para o usuário torne-se apto a realizar consultas e interagir com o ambiente.

SearchCourses: Operação destinada a realizar consultas dos cursos que o usuário esteja matriculado.

SearchClasses: Operação destinada a realizar consultas das disciplinas de um determinado curso.

SearchQuestionnaires: Operação destinada a realizar consultas dos questionários de uma ou mais disciplinas de um determinado curso.

GetQuestionnaireContent: Operação para obter o teor do questionário. Ao obter as informações é contabilizada uma tentativa válida de resposta ao questionário. Na resposta é fornecido um número da sessão do questionário utilizado para operar sobre o 
mesmo. É através deste identificador que o AVA saberá quando foi iniciada a resolução do questionário calculando o tempo de expiração do mesmo.

SubmitQuestionnaire: Realiza a submissão parcial ou total de um questionário para o AVA.

A definição dos elementos de comunicação - ou objetos de comunicação - é determinante para o sucesso da interoperabilidade entre o APP e AVA. Esses objetos são as estruturas de dados que carregam as informações necessárias para consumir e responder um WS.

A dinâmica de comunicação é realizada da seguinte forma: o MW realiza uma requisição encapsulando um objeto de comunicação que é processado pelo WS. Em seguida, o WS envia uma resposta ao MW através de um outro objeto de comunicação. Por exemplo: para consumir o serviço DoLogin o MW requisita através do objeto de comunicação LoginRequest os dados do login do usuário. Em seguida o serviço DoLogin envia a resposta com o objeto de comunicação LoginResponse.

A tabela 1 representa um exemplo da estrutura de dados de um objeto de comunicação exemplificado no formato JSON.

Tabela 1: Descrição dos atributos de comunicação.

\begin{tabular}{|c|c|c|}
\hline $\begin{array}{c}\text { Objeto de } \\
\text { Comunicação }\end{array}$ & Atributos & Exemplo (JSON) \\
\hline LoginRequest & $\begin{array}{l}\text { Login: texto } \\
\text { Password: texto }\end{array}$ & $\begin{array}{l}\text { \{"login":"joao", } \\
\text { "password":"123joao123" }\end{array}$ \\
\hline LoginResponse & $\begin{array}{l}\text { ResponseCode: inteiro } \\
\text { Log (opcional): texto } \\
\text { User: user } \\
\text { LoginDate: data } \\
\text { idSession: inteiro longo } \\
\text { ExpirationDate: data }\end{array}$ & $\begin{array}{l}\{\text { "loginDate":"Jun 30, } 2014 \text { 10:26:34 } \\
\text { AM", } \\
\text { "expirationDate":"Jun 30, } 2014 \text { 1:26:34 } \\
\text { AM", } \\
\text { "idSession":1092837465, } \\
\text { "user":\{"id":14592, } \\
\text { "name":"JoÃ£o Alves da Silva", } \\
\text { "login":"joao", } \\
\text { "email":"jsilva@ @mail.com"\}, } \\
\text { "responseCode":1, } \\
\text { "log":"Login realizado com sucesso" }\end{array}$ \\
\hline
\end{tabular}

Os objetos de comunicação definidos no modelo de interoperabilidade utilizam constantes que definem e indicam seus valores e status. Por exemplo: o usuário pode salvar as respostas do questionário para concluí-lo em outro momento ou envia-las definitivamente para o AVA. Neste primeiro caso, a constante Questionnarie_Operation_Store, com o valor 2, utilizada pelo objeto de comunicação SubmitQuestionnarieRequest, no atributo Operation, informa ao AVA que as repostas do questionário serão salvas. No segundo, a constante Questionnarie_Operation_Send, com o valor 1, utilizada pelo objeto de comunicação SubmitQuestionnarieRequest, no atributo Operation, informa que as repostas do questionário serão enviadas em definitivo para o AVA. 
Foi implementada uma biblioteca em JAVA que contém todos os objetos de comunicação, objetos de domínio, constantes e métodos auxiliares para validação dos atributos, requestes e responses. Através da biblioteca o AVA terá a facilidade de implementar seus serviços preocupando-se apenas com o processamento das informações, já que as mesmas estão padronizadas na biblioteca. Da mesma forma o APP poderá obter facilidade para criar as requisições no formato especificado no modelo e interpretar as respostas do AVA. Essa biblioteca está disponível para download através do link https:/www.dropbox.com/s/duicxayeftoj6tb/modelointeroperabilidade-v1.0.jar .

A documentação técnica do modelo de interoperabilidade, bem como da biblioteca, em formato JavaDoc, está disponível no https://www.dropbox.com/s/efdpto5ja7iat5e/documentacao-interoperabilidade-v1.0.pdf.

\section{Considerações Finais}

Este trabalho esteve voltado para criação de uma estrutura computacional que tornasse possível a interação entre dispositivos móveis e AVA, possibilitando que outras pessoas utilizem essa tecnologia em prol do seu desenvolvimento, seja como aluno, seja como profissional da educação. Além disso, pode proporcionar que outros pesquisadores utilizem o modelo proposto como referência para outros trabalhos, como também possam evoluí-lo, sugerindo melhorias e ou acrescentando novos recursos que possibilitem o uso de outras funcionalidades disponíveis nos AVA através de dispositivos móveis.

Consideramos que a realização deste trabalho trouxe como contribuição para área educacional a ampliação das possibilidades de interação entre professor e aluno, uma vez que o produto final vem favorecer o rompimento dos limites da sala de aula. Esta é mais uma iniciativa de facilitar a comunicação sem restrições de espaço e tempo, tornando a interação e consequentemente a aprendizagem situações ilimitadas.

Para a computação, podemos ressaltar o investimento no padrão de interoperabilidade deixado para a comunidade acadêmica. $\mathrm{O}$ produto criado e testado vem favorecer a comunicação efetivada por meio dos dispositivos móveis, o que traz mais uma conquista em relação ao uso da tecnologia em prol da formação humana.

Reconhecemos que este trabalho não tem a pretensão de encerrar as discussões entre o uso de aplicativos móveis para a Educação. Ele pode ser considerado um ponto de partida para investimentos futuros que permitam ainda mais a aplicação dos conhecimentos computacionais à aprendizagem, tendo em vista que a perspectiva é de maior interação entre estas áreas de grande repercussão.

Como continuidade e evolução deste trabalho, o modelo proposto poderá ser utilizado como estratégia de flipped classroom (sala de aula invertida), possibilitando que os alunos aprendam os conteúdos em qualquer ambiente por meio de recursos diferentes, antes mesmo de chegarem ao momento da aula com o professor. Os alunos farão uso do modelo para terem acesso, antes das aulas, aos conteúdos postados nos AVA. É importante que o próprio modelo de interoperabilidade possa ser evoluído e que nossas pesquisas sejam continuadas, seja através do desenvolvimento de novas 
possibilidade de comunicação com os AVA, funcionalidade que envolvam algum tipo de processamento no middleware, implementação de segurança, entre outros.

\section{Referências Bibliográficas}

Bottentuit, João Batista . (2012) Do Computador ao Tablet: Vantagens Pedagógicas na Utilização de Dispositivos Móveis na Educação. Revista Educaonline, v. 6, p. 125149, 2012.

Bowker, R.R. Wireless Trainning or " $m$-learning" is here: first movers in the. Acessado em 15 de abril de 2014.

Coulouris, George; Dollimore, Jean; Kindberg, Tim (2005).“Distributed systems: concepts and design”. 4. ed. Addison-Wesley, 2005. ISBN 0321263545

Georgieva, E.; Smrikarov, A.; Georgieva, T., (2011) "Evaluation of mobile learning system”. Procedia Computer Science, Philidelphia, v. 3, p. 632-637, 2011.

Kalin, Martin. (2009) “Java Web Services”. Alta Books. Ed.1, p. 1.

Koschembahr, C. (2005).“Mobile Learning: The Next Evolution. Chief Learning Officer", fev, 2005.

Kreger, H. (2001) “Web Services Conceptual Architecture”. IBM Software Group.

Mahmoud, Qusay H.(2004) “. Middleware for Communications”. Editora John Wiley e Sons, 2004. ISBN:0470862068.

Perrenoud, P. (2000) “10 Novas Competências para Ensinar", Artmed pool. Life long learning. Market report, p. 5-22.

Rodrigues, P.A. (2011) "iTarefa: componente Moodle para incorporar Módulos de Aprendizagem Interativa em cursos WEB”. In: 22 ${ }^{\circ}$ Simpósio Brasileiro de Informática na Educação.

Silva, L.C.M., Neto, F.M.M. e Junior, L.J. (2011) "MobiLE: Um Ambiente Multiagente de Aprendizagem Móvel para Apoiar a Recomendação Sensível ao Contexto de Objetos de Aprendizagem". In: XXII Simpósio Brasileiro de Informática na Educação, p. 254-263.

Souza, Vinícius. (2004)“SWservice: uma biblioteca para a escrita da língua brasileira de sinais baseada em web services. Dissertação de Mestrado, Programa de Pós Graduação em Computação Aplicada - Unisinos, São Leopoldo.

Yau, J.K. e Joy, M. (2010) "A Context-Aware Personalized m-Learning Application based on m-Learning Preferences". In: 6th IEEE International Conference on Wireless, Mobile and Ubiquitous Technologies in Education, p. 11-18. 